\title{
Methotrexate-induced nausea and vomiting in adolescent and young adult patients
}

\author{
Pravin Patil • Richard A. Parker • Christine Rawcliffe • \\ Abigail Olaleye • Samantha Moore • Nicola Daly • \\ Debajit Sen • Yiannis Ioannou
}

Received: 1 March 2013 /Revised: 27 August 2013 /Accepted: 3 September 2013 /Published online: 10 October 2013

(C) The Author(s) 2013. This article is published with open access at Springerlink.com

\begin{abstract}
This study aims to determine the prevalence of methotrexate-induced nausea and vomiting in both adolescent and adult patients with inflammatory arthritis. A survey of methotrexate side effects was conducted on patients with inflammatory arthritis. We provided a brief questionnaire to unselected patients with inflammatory arthritis being treated with methotrexate attending adolescent and adult rheumatology clinics. The questions related to the presence, absence, and severity of nausea and vomiting, the temporal relationship with methotrexate and whether anti-emetics had been prescribed. A total of 106 patients from the age of 13 years and above -57 adults (over 20 years) and 49 adolescents (1319 years) were included in this study. The median age for those experiencing nausea was 19 years (interquartile range (IQR) 7) and for those with no nausea 55 years (IQR 46) $(p<0.001)$. Thirty-six out of 49 adolescent patients reported nausea $(73 \%)$ compared to only $20 / 57$ adults (35\%) $(p<0.001)$. Multiple logistic regression analysis showed that the nausea group had a significantly higher proportion of adolescents $(p=0.0002)$, patients taking subcutaneous (SC) methotrexate MTX $(p=0.002)$, and patients with duration of MTX of more than 1 year $(p=0.049)$. Adolescents were
\end{abstract}

P. Patil $\cdot$ C. Rawcliffe $\cdot$ A. Olaleye $\cdot$ S. Moore $\cdot$ N. Daly $\cdot$ D. Sen $\cdot$ Y. Ioannou

Arthritis Research UK Centre for Adolescent Rheumatology, UCL Hospital and Great Ormond Street Hospital, University College London (UCL), London, UK

R. A. Parker

Centre for Applied Medical Statistics, University of Cambridge, Cambridge, UK

Y. Ioannou $(\bowtie)$

Arthritis Research UK Centre for Adolescent Rheumatology, Room 411, 4th Floor Rayne Institute, 5 University Street, London WC1E $6 \mathrm{JF}, \mathrm{UK}$

e-mail: y.ioannou@ucl.ac.uk estimated to have over 6 times higher odds of nausea compared to adults (OR 6.31, $95 \%$ CI 2.38 to $16.75, p=0.0002$ ) after adjusting for SC MTX and duration of MTX. Only $22 \%$ of adolescents and $10 \%$ of adults were prescribed antiemetics. There is a higher prevalence of MTX-induced nausea and vomiting in adolescents and younger adult patients with inflammatory arthritis compared to older adults. The role of anti-emetics in the treatment of these symptoms is unclear.

Keywords Adolescents · Inflammatory arthritis ·

Methotrexate $\cdot$ Nausea

$\begin{array}{ll}\text { Abbreviations } \\ \text { MTX } & \text { Methotrexate } \\ \text { JIA } & \text { Juvenile idiopathic arthritis } \\ \text { IQR } & \text { Interquartile range } \\ \text { SC } & \text { Subcutaneous } \\ \text { OR } & \text { Odds ratio } \\ \text { 5-HT3 } & \text { 5 Hydroxytryptamine3 }\end{array}$

Introduction

Nausea is one of the most frequent side effects of methotrexate (MTX) [1] that may adversely impact on the adherence and tolerability of this drug. These issues are especially important in the care of adolescent patients, yet little is known regarding the prevalence of these side effects in this age group, whether it differs from adult patients, to what extent and what the risk factors may be. We conducted a survey of adolescent and adult patients with inflammatory arthritis being treated with MTX to determine if MTX-induced nausea and vomiting was present and if so, whether this was being adequately treated with anti-emetics. Data was collected from a self-completed 
questionnaire of patients on methotrexate attending a single unit's clinic.

\section{Methods}

Patients with inflammatory arthritis, taking MTX, attending a dedicated adolescent rheumatology clinic and an adult rheumatology MTX monitoring clinic at University College London Hospital (UCLH) over a 12-week period were included in this study. Adolescence was defined as 10-19 years in keeping with the WHO [2]. The adolescent unit at UCLH cares for patients from age 12 years and above.

This survey was performed under good clinical practice conditions. A MTX intolerance enquiry questionnaire was constructed, consisting of two sections. The first section was designed to explore the dose/duration of methotrexate, folic acid supplementation, compliance with treatment, and side effects experienced by the patient. In this section, patients were requested to tick the side effects they were experiencing from a list of all possible methotrexate-related side effects. Only patients who ticked yes to nausea or vomiting were asked to proceed with the second part of the questionnaire which explored these symptoms in further details.

The second section obtained information regarding the onset of these symptoms - whether prior to (anticipatory), when thinking of (associative) or occurred after taking MTX. On each item, a patient could score intensity as 0 (no complaints), 1 (mild complaints), 2 (moderate complaints) or 3 (severe complaints). Duration of symptoms was scored as lasting few hours, $>1$ day and all the time. Last question was regarding the use of anti-emetics and its benefit.

For the purpose of this study, we included patients who were using either oral or parenteral MTX as a single DMARD therapy for at least 3 months. From the medical records, information was confirmed on duration of MTX use, MTX route of administration and MTX dose. A doctor or nurse personally discussed the survey and handed over the questionnaire to all the patients who visited the rheumatology clinic during the study period if they met above the criteria. There was no help required from clinical staff to complete this questionnaire as it only consisted of a set of simple tick box style questions. Younger adolescents occasionally consulted an accompanying adult to complete the questionnaire. All patients returned the completed questionnaire back to the clinical staff.

As this was a survey to determine incidence of nausea/ vomiting and anti-emetic prescribing in patients on MTX, no ethical approval was required. All questions used to collect the information were those used in routine clinical consultation as part of the patient's standard care. Nausea is difficult to measure, being a subjective symptom, and its perception can vary widely. It was therefore necessary to collect data with the help of a set of appropriate questions prompting patients to accurately report the occurrence and impact of nausea.

Statistical analysis

For categorical variables with more than two categories, a chisquared test-for-trend was used to compare between age groups (adolescent or adult). Otherwise, analysis of categorical variables involved using Fisher's exact tests to compare between groups. In particular, Fisher's exact tests were used to compare the incidence of nausea and vomiting between age groups; and this analysis was supported by the presentation of the $95 \%$ confidence interval for the difference in percentages. These $95 \%$ confidence intervals were calculated using Newcombe's method [3]. A non-parametric Mann-Whitney $U$ test was used to compare patient age between those with nausea versus those without; whereas an independent sample $t$ test was used to compare MTX dose between groups because this was an approximately normally distributed continuous variable.

A logistic regression method was used to investigate the effect of several variables at once on the incidence of nausea and vomiting. Forwards model selection (likelihood ratio testing) was used in the context of logistic regression to identify significant predictors of outcome among the variables gender, MTX dose, subcutaneous (SC) MTX, duration, and age group (adolescent or adult). Results were presented as odds ratios (OR) with $95 \%$ confidence intervals.

All analyses were computed using SPSS software version 18 [4], except $\mathrm{R}$ software [5] was used to calculate $95 \%$ confidence intervals for the difference in proportions and Prism used to calculate Mann-Whitney U. A $5 \%$ significance level was used throughout.

\section{Results}

We surveyed a total of 106 patients - 57 adults ( $68 \%$ females) and 49 adolescents (71\% females). The median age of the adults was 55 years (interquartile range (IQR) 28) and the adolescents 18 years (IQR 2). Thirty-three percent of adults were on SC MTX (19/57) compared to $49 \%$ of adolescents (24/49). Between the two age groups, there was no significant difference in gender $(p=0.83)$ or dose of MTX $(p=0.12)$. Combing all the patients, the median age for presence of nausea was 19 years (IQR 7) and those with no nausea 55 years (IQR 46) $(p<0.001)$ [Fig. 1]. Thirty-six out of 49 adolescents reported nausea ( $73 \%$ ) compared to only $20 / 57$ adults $(35 \%)$ $(p<0.001)$. Twenty-one out of 49 adolescents experienced vomiting (43\%) compared to only 6/57 adults (11\%) $(p<$ $0.001)$. Interestingly, patients on parenteral MTX reported higher prevalence of nausea compared to those on oral MTX (77 versus $37 \%, p<0.0001$ ). Bivariate analyses (Table 1) 


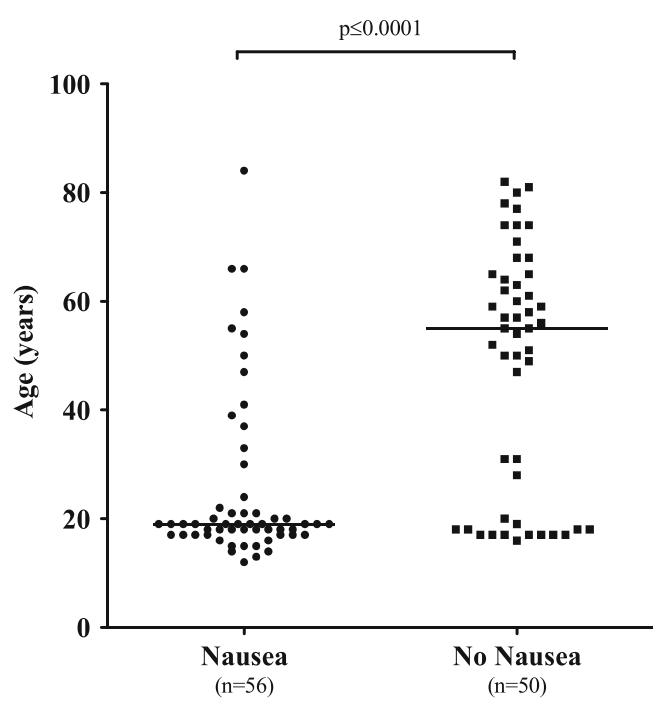

Fig. 1 Patients with inflammatory arthritis taking methotrexate attending adolescent and adult rheumatology clinics. Median is shown for each and comparison made using Mann-Whitney $U$ test

showed that the nausea group had a significantly higher proportion of adolescents $(p<0.0001)$, females $(p=0.019)$, patients taking SC MTX $(p<0.0001)$, and patients with duration of MTX of more than 1 year $(p=0.04)$. The group in those with vomiting also had a significantly higher proportion of adolescents $(p=0.0002)$. There was insufficient evidence that MTX dosage is associated with either nausea or vomiting.

Separate multiple logistic regression analyses were performed on the nausea and vomiting outcome variables, using forwards model selection (likelihood ratio testing) to identify significant predictors of outcome. Gender, MTX dose, SC MTX, duration of MTX, and age group (adolescent or adult) were all made available for inclusion in the logistic regression models. The final model results for nausea are shown in Table 2.

Adolescent patients were estimated to have over 6 times higher odds of nausea compared to adult patients (OR 6.31, $95 \%$ CI 2.38 to $16.75, p=0.0002$ ) after adjusting for SC MTX and duration of MTX. SC MTX (OR 4.49, $95 \%$ CI 1.71 to $11.79, p=0.002$ ) and duration of MTX (OR 3.86, $95 \%$ CI 1.00 to $14.89, p=0.05$ ) were also found to be significantly associated with nausea. Although the bivariate analyses suggested gender as a possible predictor of outcome, there was no significant effect of gender on nausea after adjusting for age group, SC MTX and duration. Only the age group was a significant predictor of vomiting. Again, adolescent patients were estimated to have over 6 times higher odds of vomiting compared to adult patients (OR 6.38, $95 \%$ CI 2.30 to 17.64; $p=0.0004)$.

In both groups, nearly all patients who reported nausea and vomiting were experiencing these symptoms after methotrexate intake. Fifty two percent (19) of adolescents and $30 \%$ [6] of adults had associative nausea. While none of the adult patients experienced anticipatory or associative vomiting,

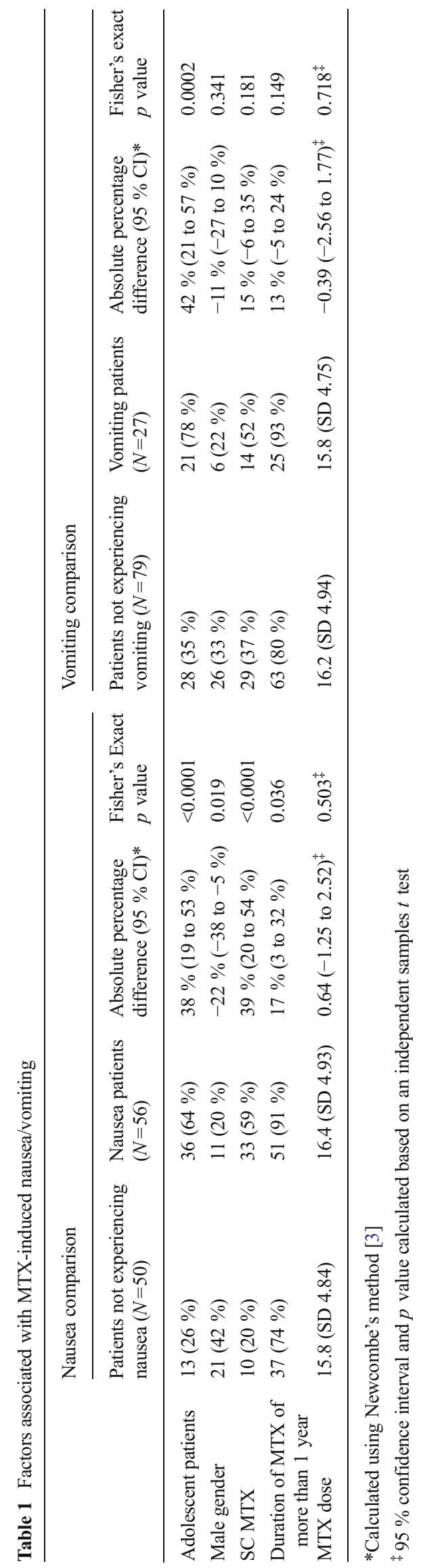


Table 2 Multiple logistic regression analysis: performed using forward model selection with a $5 \%$ significance level

\begin{tabular}{llll}
\hline & Odds ratio & $95 \%$ CI & $p$ Value \\
\hline Adolescent & 6.312 & 2.379 to 16.746 & 0.0002 \\
SC MTX & 4.487 & 1.708 to 11.785 & 0.002 \\
$\begin{array}{l}\text { Duration of MTX more } \\
\text { than 1 year }\end{array}$ & 3.865 & 1.003 to 14.889 & 0.049 \\
\hline
\end{tabular}

The outcome is nausea (yes or no)

$13 \%$ of adolescents reported these symptoms. Forty one percent (15) of adolescents and 30\% [6] of adults experienced anticipatory nausea.

Only $11(22 \%)$ adolescents and $6(10 \%)$ adults indicated being on anti-emetics. Six adolescents (55\%) and five adults $(83 \%)$ reported none or little benefit from anti-emetics. Only one adult experienced "quite a lot" of benefit.

\section{Discussion}

To our knowledge, this is the first study to demonstrate a clear inverse relationship between the presence of MTX-induced nausea/vomiting and age in adolescent and adult patients. A high prevalence of MTX-induced gastro-intestinal intolerance in JIA has recently been reported by Bulatović et al. [6]. Our questionnaire contained similar information as to the nausea and vomiting domain of MTX intolerance severity score questionnaire used by Bulatović et al.

We found a higher prevalence of nausea and vomiting not only in adolescents but also in young adults showing a clear inverse relationship between MTX-induced nausea and age. As demonstrated in Table 1, there was insufficient evidence of a relationship between nausea and dose of MTX, which was unexpected. This is supported by a study by Lin et al. which reported that there were no significant differences between MTX concentrations above or below safety values and nausea/vomiting in adult oncology patients on high-dose methotrexate chemotherapy [7]. It is still unknown which factors influence the MTX metabolic process, and the effects, if any, of age and sex. In our study, the association of nausea in patients on MTX for more than 1 year raises the possibility of a cumulative effect of this drug on nausea.

Higher prevalence in patients on parenteral MTX may be related to the frequent occurrence of anticipatory and associative complaints in adolescents. It suggests that classical conditioning plays an important role in MTX intolerance in this group $[6,8,9]$. However, it is interesting to note that Verkaaik et al. did not find behavioural therapy and parenteral MTX being more effective than the oral MTX with anti-emetics in treating MTX intolerance [10]. Whilst the exact cause of this complex issue in younger patients with inflammatory arthritis is unknown, stimulation of chemoreceptor trigger zone has been postulated. Therefore, treatment with 5-HT3 receptor antagonist has been suggested by some authors [11, 12]. In our study, very few patients were taking anti-emetics. It would be interesting to study this further with a view of finding out compliance with anti-emetics and reasons for poor adherence.

Although our study has a moderate sample size for a study of adolescent patients with arthritis, we accept that there may have been low power to detect clinically relevant effects due to the overall small sample size. Also, there are some limitations given that this is a self-reported data. In particular, respondent bias may have affected the results. Also, as a service evaluation study rather than a research study, it might not have captured all factors associated with MTX-induced nausea and vomiting. For example, it would be relevant to have also included body mass index in the multiple regression analysis, however, this data was unavailable given that height is not routinely measured in the adult clinic. We observed that patients on parenteral MTX had a higher prevalence of nausea/vomiting. It is possible that the same patients had these symptoms whilst on oral MTX and hence, they were switched to parenteral form. Findings presented here should be considered as exploratory rather than confirmatory. It is not possible to identify causal relationships on the basis of a cross-sectional study. It would also be of interest to investigate younger adolescents and paediatric patients to ascertain if these findings indeed peak with adolescence or are also as or more prevalent in younger children.

In conclusion, we have found evidence of a higher prevalence of MTX-induced nausea and vomiting in adolescents and younger adult patients with inflammatory arthritis compared to older adults. In addition to younger age, these symptoms are associated with parenteral administration and longer duration of MTX. The usefulness of anti-emetics in the treatment is unclear. These findings support a more proactive approach to counselling and managing MTX-induced nausea in adolescent and young adult patients with inflammatory arthritis. This may in part explain why adherence is often a particular problem in younger patients. Further scientific study is required to delineate why younger age associates with more nausea.

Acknowledgments The authors are especially grateful to all patients, parents, and their referring physicians. YI is supported through an Arthritis Research UK Grant (ref:20164) and is also supported by the National Institute for Health Research University College London Hospitals Biomedical Research Centre.

Disclosures None.

Open Access This article is distributed under the terms of the Creative Commons Attribution License which permits any use, distribution, and reproduction in any medium, provided the original author(s) and the source are credited. 


\section{References}

1. Dhir V, Aggarwal A (2012) Methotrexate-related minor adverse effects in rheumatoid arthritis: more than a nuisance. J Clin Rheumatol : Pract Rep Rheum Musculoskelet Dis 18(1):44-46

2. WHO | Adolescent health [Internet]. World Health Organization. Available from: http://www.who.int/topics/adolescent health/en/. Accessed 14 Feb 2013

3. Newcombe RG, Altman DG (2000) Proportions and their differences. In: Altman D, Machin D, Bryant D, Gardner S (eds) Statistics with confidence: confidence intervals and statistical guidelines, 2nd edn. BMJ Books, London

4. SPSS/PASW for Windows, Rel. 18.0.3. 2010. Chicago: SPSS Inc.

5. R Development Core Team (2012) R: A language and environment for statistical computing. R Foundation for Statistical Computing, Vienna, Austria. ISBN 3-900051-07-0 www.r-project.org

6. Bulatović M, Heijstek MW, Verkaaik M, Van Dijkhuizen EHP, Armbrust W, Hoppenreijs EPA et al (2011) High prevalence of methotrexate intolerance in juvenile idiopathic arthritis: development and validation of a methotrexate intolerance severity score. Arthritis Rheum 63(7):2007-2013

7. Lin F, Juan Y, Zheng S-E, Shen Z, Tang L-N, Zhao H et al (2009) Relationship of serum methotrexate concentration in high-dose meth- otrexate chemotherapy to prognosis and tolerability: a prospective cohort study in Chinese adults with osteosarcoma. Current Therapeutic Research. Excerpta Med Inc 70(2):150-160, All rights reserved

8. Van der Meer A, Wulffraat NM, Prakken BJ, Gijsbers B, Rademaker CMA, Sinnema G (2007) Psychological side effects of MTX treatment in juvenile idiopathic arthritis: a pilot study. Clin Exp Rheumatol 25(3):480-485

9. Figueroa-Moseley C, Jean-Pierre P, Roscoe JA, Ryan JL, Kohli S, Palesh OG et al (2007) Behavioral interventions in treating anticipatory nausea and vomiting. J Natl Compr Cancer Netw : JNCCN 5(1): 44-50

10. Verkaaik M, Bulatović M, Sinnema G, Rademaker C, Wulffraat N (2011) Treatment of methotrexate (MTX) intolerance: behavioural therapy, versus switch to parenteral MTX versus oral MTX. Pediatr Rheumatol BioMed Central Ltd 9(Suppl 1): $\mathrm{O} 23$

11. Kempinska A, Benchimol EI, Mack A, Barkey J, Boland M, Mack DR (2011) Short-course ondansetron for the prevention of methotrexate-induced nausea in children with Crohn disease. J Pediatr Gastroenterol Nutr 53(4):389-393

12. Blanco R, González-Gay MA, García-Porrúa C, Ibañez D, GarcíaPais MJ, Sánchez-Andrade A et al (1998) Ondansetron prevents refractory and severe methotrexate-induced nausea in rheumatoid arthritis. Br J Rheumatol 37(5):590-592 